\title{
Some Properties DNA from the Point of View the Quantum Information
}

\author{
Mehdi Mirzaee ${ }^{1, *}$, Milad Falahat Chian ${ }^{2}$ \\ ${ }^{1}$ Department of Physics, Arak University, Arak, Iran \\ ${ }^{2}$ Department of Biology, Arak University, Arak, Iran \\ *Corresponding Author: m-mirzaee@araku.ac.ir
}

Copyright (C)2013 Horizon Research Publishing All rights reserved.

\begin{abstract}
Molecules are the basis of life. They can consider as a quantum system. So it is necessary to enter quantum mechanics in biological discussion. Specifically, we should expected the quantum to be the basis for heredity, genetic, biology and DNA. In this paper we consider the DNA molecules as a quantum system. We discus about the relation between the heredity, biology and DNA and quantum systems. We also claim that there is a relation between the quantum information and some biologicaly-process such as cell division, cloning so that we think that the root of all problems in cloning may be in the quantum effects.
\end{abstract}

Keywords Quantum Information, DNA, Genetic, Molecular Biology, Biophysics

Pacs: 03.67.2a; 82.39.Pj

\section{Introduction}

Cloning, or asexual reproduction, a time-honored method of reproduction, is the method by which many extinct and existing species of organisms reproduced [34]. Cloning research has been underway since the 1890. In July of 1996, Scottish scientists created the first animal cloned from an adult cell. On July 5, 1996, Dolly the sheep was born at the Roslin Institute in Edinburgh Scotland. The announcement of her birth in early 1997 shocked the scientific community and stirred debate over the possibility of cloning humans. A process known as cell nuclear replacement created Dolly by transferring a mammary cell of a six-year-old white Welsh Mountain sheep into the egg cell of a Scottish Blackface ewe. But it was the groups creation of Dolly, cloned from an adult udder cell, that finally overturned the idea that in mammals, developed cells could not reverse their fate[4]. After Dolly, scientists thought they would soon be able to clone many other mammals including: mice, cattle, sheep, pigs, goats, rabbits and a cat. It is clear that there are potentially great benefits resulting from the concept of cloning, such as development of identical animals for biomedical research or for production of human proteins. However, there are also serious risks that come with the process. The low efficiency of the cloning process has stymied industrial development, and most companies set up to commercialize cloning have shut down [4]. Cloned animals have unpredictable problems with gene expression [5]. Animals with the same genotype do not have the same phenotype. This problem is heightened with embryonic stem cells. This problem is neither predictable and can be triggered throughout the life of the cloned organism. This is because of gene regulation by DNA metylaltion, which is a kind of memory of the cells. Only 25 percent into healthy offspring. This is slightly better than a decade ago Dolly was the only lamb born from 277 cloned embryos but it is still far below the efficiency demanded by industry. The telomeres of Dolly were abnormally short, suggesting that Dolly was prematurely aged at birth. Dolly gave birth to two normal lambs after two pregnancies. These lambs have normal telomeres. All the cloned animals born after Dolly that were examined have essentially normal telomeres. Several clones obtained from a 17-year-old bull have normal telomeres. It is currently impossible to correlate early arthritis in Dolly with the length of her telomeres. Many scientist thought that there is a direct relationship between the size of telomeres in animal cloned and abnormality. But this phenomenon may not be related to cloning or may result from an ageing process indirectly linked to telomere length. Clones must therefore be considered as epigenetically modified animals. It is clear that all cloned animals are biologically flawed [7], also the cause of abnormal cloned have remained unknown although a cloned stem cell is perfectly normal. On the other hand the size of telomere as a result of visual effect is not result of abnormality. On the other hand there is growing thought among physicists $[8,9,10,11,12]$ and biologists [13] that quantum mechanics might play an important role in living systems. McFadden has the view that ultimately the origin of life will be answered by quantum mechanics [13]. To cite a few others, Frolich has suggested that the action of enzymes in biological systems would be understood from quantum mechanical principles [14]. Home and Chattopadhyaya have proposed a way to describe quantum mechanical measurement pro- 
cess in a DNA molecule, which may be in a superposition of mutational states [35]. McFadden and Al-Khalili have argued that during cell mutation quantum mechanics may play an important role and entanglement between the mutational state and the environment may enhance the probability of mutation [18]. To be sure, biological systems are quintessential information processors. Patel has proposed that a quantum algorithm may be at work in genetic evolution [36], though skepticism prevails on how quantum coherence can be maintained in a DNA molecule. Profesor Anton Zeilinger's group in Vienna [17] have recently demonstrated that the fullerene molecule, composed of 60 carbon atoms (the famous 'buckyball'), can pass through two slits simultaneously. Fullerene molecules are spheres with a diameter similar to that of the DNA double helix. If fullerene can enter the quantum multiverse then DNA may do the same.

At this stage we think that the root of this problems may be in the quantum effects not in classical ones. So that the quantum behaviour can arise in some circumstances in biology $[20,21,22]$. It is therefore worth studying biomolecules as quantum mesoscopic systems. At this aim, we concentrate on one of the essential features of information: the possibility to copy it. One might think that this possibility is an essential feature of any good encoding of information. This is however not the case: when information is encoded in quantum systems, in general it cannot be replicated without introducing errors. So that this errors is cause of all abnormality in animal cloned.

The paper is organized as follows; In section-2 we define the cloning and the characteristics and structure of cloning. In section-3 we discuss about the potential medical benefits of animal cloning. In section- 4 we discuss about abnormality and problems in animal cloning. In section-5 we define telomere and telomeras. In section6 we pay to quantum theory as a answer to the above unsolved problems. Finally, the connection between the quantum theory (information) and problems in animal cloned is discussed.

\section{What is the cloning}

Cloning is the process of producing genetically identical organisms $[2,3]$. It may involve division of a single embryo, in which case both the nuclear genes and the small number of mitochondrial genes would be identical, or it may involve nuclear transfer, in which case only the nuclear genes would be identical.

It is inherent in the process of sexual reproduction that the progeny differ genetically from one another. In contrast, asexual reproduction (cloning) produces genetically identical progeny. This is a common form of reproduction in plants, both in nature and in the hands of plant breeders and horticulturists. Once a desired combination of characteristics has been achieved, asexual reproduction is the best way of preserving it. Asexual reproduction is also common among some invertebrate animals (worms, insects). Asexual reproduction in plants and invertebrates usually takes place by budding or splitting [34].

The first successful cloning in vertebrate animals was reported in 1952, in frogs. Nuclei from early frog embryos were transferred to unfertilized frog eggs from which the original nuclei had been removed. The resulting clones were not reared beyond the tadpole stage. In the 1960s, clones of adult frogs were produced by transfer not only of nuclei from early embryos but also of nuclei from differentiated larval intestinal cells. Later, clones of feeding tadpoles were obtained by nuclear transfer from differentiated adult cells, establishing that differentiation of cells involving selective gene expression does not require the loss or irreversible inactivation of genes. Nuclear transfer in frogs has not yet generated an adult animal from cells of an adult animal.

Nuclear transfer can be used for different objectives. Nuclear transfer in mice has been used to show that both a female and a male set of genes are required for development to birth. If the two pronuclei, taken from fertilized eggs and transferred into an enucleated egg, are only maternal or only paternal, normal development does not occur. This is not cloning, since the single embryo formed is not identical to any other embryo and the objective is not to multiply individuals.

Nuclear transfer has also been used for cloning in various mammalian species (mice, rabbits, sheep, cattle), but until recently only nuclei taken from very early embryos were effective, and development was often abnormal, for reasons that are not fully understood.

In 1996, a new method of cloning sheep embryos was reported, which involved first establishing cell cultures from single embryos. Nuclei from the cultured cells were transferred to enucleated unfertilized sheep eggs, particular attention being paid to the cell cycle stage of both donor and host cells, and the eggs were then artificially stimulated to develop. Genetically identical normal lambs were born.

Cell cultures were then established not only from embryonic and fetal stages, but also from mammary tissue taken from a 6-year-old sheep. Nuclear transfer was carried out as before, and in 1997 it was reported that several lambs had been born from the embryonic and fetal transfers and one lamb named Dolly (out of 277 attempts) from the adult nuclear transfer. It is not known whether the transferred nucleus was from a differentiated mammary gland cell or from a stem cell.

From the point of view of basic research, this result is important. If repeatable it may allow greater insight into the aging process, how much is due to cell aging, and whether or not it is reversible. Such work may also increase our understanding of cell commitment, the origin of the cancer process, and whether it can be reversed, but at the present time the research is at a very early stage. Dolly may have a shortened life span or a greater susceptibility to cancer: if she is fertile, her progeny may show an increased abnormality rate, owing to the accumulation of somatic mutations and chromosomal damage.

\section{The potential uses of cloning animals}

Potential uses of cloning animals are reported to include:in the field of medicine and medical research, to 
improve genetic and physiological knowledge, to make models for human diseases, to produce at lower cost proteins like milk proteins to be used for therapeutic aims, to provide a source of organs or tissues for xenotransplantation;

in agriculture and agronomical research, to improve the selection of animals or to reproduce animals having specific qualities (longevity, resistance, . . . ) either innate, or acquired by transgenesis.

From the point of view of animal breeding, the technology could be useful, in particular if it increases the medical and agricultural benefits expected from transgenesis (genetic modification of animals). By using genetic modification and selection in cultured cell lines, rather than in adult animals, it could become possible to remove genes, such as those provoking allergic reactions, as well as adding genes, for the benefits of human health.

\section{Abnormality in cloned animals}

A particular point was the topic of discussion after the birth of Dolly. The telomeres of this animal were abnormally short, suggesting that Dolly was prematurely aged at birth. Dolly gave birth to two normal lambs after two pregnancies. These lambs have normal telomeres. All the cloned animals born after Dolly that were examined have essentially normal telomeres. Several clones obtained from a 17-year-old bull have normal telomeres. This suggests that cloning per se does not induce a shortening of telomeres. In fact, Dolly's telomeres were shorter than normal because the telomeres of the cells used as nuclear donors were already short before cloning. These cells were obtained from a six-year-old sheep, but more importantly they were cultured for long periods of time. Interestingly, the telomeres of Dolly's genome reached normal length in offspring. Sexual reproduction is thus able to restore damaged telomeres. This event probably occurred during gametogenesis. It is currently impossible to correlate early arthritis in Dolly with the length of her telomeres. This phenomenon may not be related to cloning or may result from an ageing process indirectly linked to telomere length. Clones must therefore be considered as epigenetically modified animals. It is striking that the capacity of the cells to give birth to cloned animals after nuclear transfer decreases as the number of passages increases. Little is known of the events that induce this phenomenon. They seem to be related to culture conditions. Many studies are obviously required to understand these problems [1, 41]. An empirical approach is currently expected to find the best culture conditions for improving cloning efficiency and for defining the experimental situations in which cells keep or lose their capacity to give rise to cloned animals $[2]$.

Only 25 percent of cloned animal embryos grow into healthy offspring. This is slightly better than a decade ago Dolly was the only lamb born from 277 cloned embryos but it is still far below the efficiency demanded by industry $[4,6]$.

The failure of researchers to find a single mouse in which the six selected genes were all functioning normally indicated to researchers that even if clones ap- pear healthy they might have abnormal gene expression.Despite the success in the production of Dolly, animal cloning has a high rate of failure. On average, only one to two percent of the reconstructed eggs lead to live births.28 Prior to the successful birth of Dolly, researchers at the Roslin Institute produced over 200 cloned sheep embryos [23]. For every successful clone that is born, many other cloned animals die of mysterious causes [24]. The unpredictability of cloning seems to lie in unstable genes [25]. Researchers in Scotland32 believe a possible cause for the failures associated with cloning may be the result of the clones DNA missing a few carbon atoms. When sheep embryos are manipulated, they can lose some of the methyl groups attached to their genes. This change, termed imprinting, alters how actively the genes produce proteins that are key to survival. Researchers at the Massachusetts Institute of Technology looked at six imprinted genes of cloned mice [25]. They found no cloned mouse that had all six genes functioning normally. The genetic instability appears to be a random process and the developmental abnormalities are the result of many malfunctions. Less than ten percent of cloned embryos survive [26]. Many of the cloned offspring die during the late stages of pregnancy or soon after birth. Those that do survive are prone to health problems. In the early experiments at the Roslin Institute, 42 percent of the cloned lambs died within a few days of birth [27]. Many of the cloned cattle and sheep that survive to birth are born much larger in size than normal offspring. One of a pair of cloned bantengs had to be euthanized because it was abnormally large. The calf weighed almost twice as much as a normal banteng calf. Despite its size, the calf appeared healthy at first. The veterinarians at the San Diego Zoo decided to euthanize it for humane reasons. Additionally, many clones have abnormally large placentas. Cloned mice tend to be obese [28]. Clones may also be less intelligent than their naturally conceived counterparts. It has been claimed that cloned mice learn slower than normal mice. Newborn cow clones scored lower on average than nonclones on tests of attentiveness and intelligence. Other problems that clones may suffer include: low blood oxygen levels, high carbon dioxide levels, enlarged tongues, enlarged hearts, squashed faces, subfunctional kidneys, intestinal defects, diabetes, and shortened tendons that disfigure the animals feet and make them useless. The clones are not the only ones in danger. In a single study, four in twelve surrogate mothers died from pregnancy complications. The most famous of all clones, Dolly, also suffered from health problems. Early in her life she was overweight. In 1999, it was discovered that her telomeres were twenty percent shorter than normal for a sheep of her age [29]. This led to speculation that her biological age might equal the combined age of her and her mother [29]. She was diagnosed with arthritis in January of 2002. At six and a half years of age, Dolly was euthanized [29]. Dolly was suffering from lung cancer caused by a virus. Dollys early death sparked controversy, raising questions as to what could properly be judged as her true age and the risks of premature ageing in clones. Attempts to clone primates have been unsuccessful to date. Cloned monkey cells have shown abnormal division. Cloned monkey cells have not 
been capable of replicating their genetic material accurately.65 The chromosomes do not split properly and from the first cell division the cells develop inappropriately. The resulting monkey embryos do not have the correct number of chromosomes and are lacking essential proteins. Despite the failure to clone primates so far, it is believed that at some point in the future they will be cloned [30, 31, 32, 33].

The importance of nuclear transfer studies was evident from the work on amphibian embryos by Briggs and King (1950s) and Gurdon (1960s), and on mammals by Illmensee and Hoppe (1970s1980s) and McGrath and Solter (1980s). Until recently, however, only nuclei taken from very early embryos were effective (although this procedure is basically a nucleuscytoplasm reshuffling because of the similarity between the ooplasm and the early embryonic cytoplasm). Clone development was still often abnormal, for reasons that are not fully understood $[34,33]$.

Clone embryos reimplanted in vivo, immediately or shortly after microsurgery (two-cell and eight-cell stages), exhibit the same anomalies. This is based on the assumption that the genital tract is not hostile to clone embryo development and the clone is a real embryo. Although differences in gene expression profiles most probably exist between embryos produced in vivo, in vitro, or by nuclear transplantation, developmental aberrations are much more common in animals produced by nuclear transplantation compared to those produced using standard procedures for in vitro oocyte maturation, in vitro fertilization, and in vitro embryo culture $[34,33]$.

\section{$5 \quad$ Telomere and telomeras}

Structure and function of Telomeres Telomeres are repetitive DNA sequences at the ends of all human chromosomes. They contain thousands of repeats of the six nucleotide sequence, TTAGGG and In humans there are 46 chromosomes and thus 92 telomeres (one at each end). In humans, telomeric repeats average 5-15 kilo bases. Telomere specific proteins, eg. TRF1 and TRF2 bind to the repeat sequence and protect the ends, Without these proteins, telomeres are acted upon by DNA repair pathways leading to chromosomal fusions. Telomeres are also thought to be the clock that regulates how many times an individual cell can divide [37]. Telomeric sequences shorten each time the DNA replicates. Telomeres are elements at the ends of eukaryotic chromosomes they:

1- Protect chromosomes from end- to- end fusion.

2- Prevent homologous recombination and inappropriate DNA- repair.

3- Have a role in chromosome organization at meiosis, mitosis and at interphase.

4- Reverse the progressive degradation of chromosome termini caused by the incomplete replication of the 5 end of the DNA double helix in S-phase of the cell cycle.

Structure and function of Telomerase Telomerase works by adding back telomeric DNA to the ends of chromosomes, thus compensating for the loss of telomeres, thus compensating for the loss of telomeres that normally occurs as cells divide. Most normal cells do not have this enzyme and thus they lose telomeres with each division. The telomerase control gene has been mapped to 3p21 (chromosome 3, the p (short) arm, locus 21). Although the gene for telomerase is present in all cells, In humans, telomerase is active in germ cells, in vitro immortalized cells, the vast majority of cancer cells and, possibly, in some stem cells. High telomerase activity exists in germ cells, stem cells, epidermal skin cells, follicular hair cells, and cancer cells. Research also shows that the counter that controls the wasting away of the telomere can be turned on and turned off. The control button appears to be an enzyme called telomerase which can rejuvenate the telomere and allow the cell to divide endlessly. Most cells of the body contain telomerase but it is in the off position so that the cell is mortal and eventually dies. Some cell are immortal because their telomerase is switched on, Examples of immortal cells: blood cells and cancer cells. Cancer cells do not ago because they produce telomerase, which keeps the telomere intact. The next slide show cells stained to visualize the presence of telomerase. The bottom dish was treated to produce active telomerase and is still dividing. The top dish of normal cells of the same age has stopped dividing. Telomerase and Aging Because there is very little telomerase in somatic tissues, older people have shorter telomeres, particularly in actively regenerating tissues such as skin or intestinal ephithelia. This has generated a lot of interest in the possibility that telomere length could be tied to aging. After a certain number of cell divisions, the telomeres would be so short as to somehow prevent the cell from further proliferation- putting it in a state called senescence. In other words, he proposed that telomere length offered a clock for telling a cells longevity. Scientific American. Many experiments have shown that there is a direct relationship between telomeres and aging, and that telomerase has the ability to prolong life and cell division. It is believed that shortened telomeres in mitotic (dividing) cells may be responsible for some of the changes we associate with normal aging. Healthy human cells are mortal because they can divide only a finite number of times, growing older each time they divide. Thus cells in an elderly person are much older than cells in an infant. Once the telomere shrinks to a certain level, the cell can no longer divide. Its metabolism slows down, it ages, and dies. It has been proposed that telomere shortening may be a molecular clock mechanism that counts the number of times a cell has divided and when telomeres are short, cellular senescence (growth arrest) occurs. Telomerase and cancer There is experimental evidence from hundreds of independent laboratories that telomerase activity is present in almost all human tumors but not in tissues adjacent to the tumors. Thus, clinical telomerase research is currently focused on the development of methods for the accurate diagnosis of cancer and on novel anti-telomerase cancer therapeutics [37]. The presence of telomerase in cancer cells allows them to maintain telomere length while they proliferate. In most somatic tissues, telomerase is expressed at very low levels or not at all, as cells divide, telomeres shorten. What are the Risks of Cloning? When we hear of cloning successes, we learn about only the 
few attempts that worked. What we don't see are the many, many cloning experiments that failed! And even In the successful clones, problems tend to arise later, during the animal's development to adulthood. Cloning animals shows us what might happen if we try to clone humans. What have these animals taught us about the risks of cloning?

1- High failure rate Cloning animals through somatic cell nuclear transfer is simply inefficient. The success rate ranges from 0.1 percent to 3 percent, which means that for every 1000 tries, only one to 30 clones are made. Or you can look at It as 970 to 999 failures in 1000 tries. That's a lot of effort with only a speck of a return! Why is this? Here are some reasons:

* The enucleated egg and the transferred nucleus may not be compatible

* An egg with a newly transferred nucleus may not begin to divide or develop properly

* Implantation of the embryo into the surrogate mother might fail

* The pregnancy itself might fail

2- Problems during later development Cloned animals that do survive tend to be much bigger at birth than their natural counterparts. Scientists call this "Large Offspring Syndrome" (LOS). Clones with LOS have abnormally large organs. This can lead to breathing, blood flow and other problems, Because LOS doesn't always occur, scientists cannot reliably predict whether it will happen In any given clone. Also, some clones without LOS have developed kidney or brain malformations and impaired immune systems, which can cause problems later in life.

3- Abnormal gene expression patterns Are the surviving clones really clones? The clones look like the originals, and their DNA sequences are identical. But will the clone express the right genes at the right time? In Click and Clone, we saw that one challenge is to re-program the transferred nucleus to behave as though it belongs in a very early embryonic cell. This mimics natural development, which starts when a sperm fertilizes an egg. In a naturally-created embryo, the DNA is programmed to express a certain set of genes. Later on, as the embryonic cells begin to differentiate, the program changes. For every type of differentiated cell - skin, blood, bone or nerve, for example - this program is different. In cloning, the transferred nucleus doesn't have the same program as a natural embryo, It is up to the scientist to reprogram the nucleus, like teaching an old dog new tricks, Complete reprogramming is needed for normal or near-normal development. Incomplete programming will cause the embryo to develop abnormally or fail.

4- Telomeric differences As cells divide, their chromosomes get shorter. This is because the DNA sequences at both ends of a chromosome, called telomeres, shrink in length every time the DNA is copied. The older the animal Is, the shorter its telomeres will be, because the cells have divided many, many times. This is a natural part of aging, So, what happens to the clone if its transferred nucleus is already pretty old? Will the shortened telomeres affect its development or lifespan? When scientists looked at the telomere lengths of cloned animals, they found no clear answers. Chromosomes from cloned cattle or mice had longer telomeres than normal. These cells showed other signs of youth and seemed to have an extended lifespan compared with cells from a naturally conceived cow. On the other hand, Dolly the sheep's chromosomes had shorter telomere lengths than normal. This means that Dolly's cells were aging faster than the cells from a normal sheep, To date, scientists aren't sure why cloned animals show differences in telomere length.

From the above statements, we see that all cloned animals are biologically flawed, also the cause of abnormal cloned have remained unknown. On the other hand the size of telomere as a result of visual effect is not result of abnormality. At this stage we think that the root of this problems may be in the quantum effects not in classical ones. Then it need to learn more about the quantum.

\section{What is the quantum theory}

Quantum mechanics is a fundamental branch of physics with wide applications in experimental physics and theoretical physics that replaces classical mechanics and classical electromagnetism at the atomic and subatomic levels. Quantum mechanics is a more fundamental theory than Newtonian mechanics and classical electromagnetism, in the sense that it provides accurate and precise descriptions for many phenomena that these "classical" theories cannot explain on the atomic and subatomic level. The history of quantum mechanics began essentially with the 1838 discovery of cathode rays by Michael Faraday. In 1905, Albert Einstein published his paper to explain the photoelectric effect, which proposed that light traveled as discrete bundles of energy which later come to be called photons. On the other hand light functions as both a particle and a wave, depending on how the experiment is conducted and when observations are made. The question of whether such duality also showed up in matter was tackled by the bold de Broglie hypothesis, which extended Einstein's work to relate the observed wavelength of matter to its momentum. Experiments confirmed the hypothesis in 1927, resulting in a 1929 Nobel Prize for de Broglie. Just like light, it seemed that matter exhibited both wave and particle properties under the right circumstances. Obviously, massive objects exhibit very small wavelengths, so small in fact that it's rather pointless to think of them in a wave fashion. But for small objects, the wavelength can be observable and significant, as attested to by the double slit experiment with electrons.

On the other hand we contend that at the scale of interacting molecules in the cell we must deal with the influence of quantum effects in relation to materials, energy and information. Many people may view quantum effects as mere noise. However, we would wish to qualify such a position and will argue that quantum level information is being processed in biological systems. The concept of information is shaping our world: communication, economy, sociology, statistics and etc,. During the last decade or so, information entered physics from all sides: from cosmology (e.g. entropy of blackholes1) to quantum physics (the entire field of quantum information processing). Information may take different physical forms so that there is no information without a physical carrier, and no computation without a phys- 
ical process for example DNA is the physical carrier of inheritance. Information is physical, and governed by the laws of physics and Our best framework for physical theories is quantum mechanics.

In this review, we concentrate on one of the essential features of information: the possibility to copy it. One might think that this possibility is an essential feature of any good encoding of information. This is however not the case: when information is encoded in quantum systems, in general it cannot be replicated without introducing errors. This means that for two separate systems A and B the results of an arbitrary measurement of one of the systems cannot be always equal to the result of the same measurement made with another system [40].

No-cloning theorem is one of the most fundamental theorems in quantum mechanics and in quantum computation and quantum information. Wootters and Zurek [38] in 1982 realized that, if one can build a cloning machine that produces several clones of the horizontaland vertical-polarization states of an incoming photon, then circularly-polarized states cannot yield circularlypolarized clones. The no-cloning theorem may be considered an obstacle in quantum computation, where it would be desirable to store a copy in a safe place. It is not possible to acquire information that distinguishes between nonorthogonal quantum states without disturbing the states. Suppose, more generally, that $|\psi\rangle$ and $|\phi\rangle$ are two nonorthogonal states in $\mathcal{H}(\langle\psi \mid \phi\rangle \neq 0)$ and that a unitary transformation $\mathrm{U}$ is applied to $\mathcal{H} \otimes \mathcal{H}_{E}$ (where $\mathcal{H}_{E}$ is a Hilbert space accessible to Eve) that leaves both $|\psi\rangle$ and $|\phi\rangle$ undisturbed. Then

$$
\begin{aligned}
& U:|\psi\rangle \otimes|0\rangle_{E} \longrightarrow|\psi\rangle \otimes|e\rangle_{E} \\
& |\phi\rangle \otimes|0\rangle_{E} \quad \longrightarrow \quad|\phi\rangle \otimes|f\rangle_{E}
\end{aligned}
$$

and unitarity implies that

$$
\begin{gathered}
\langle\psi \mid \phi\rangle=\left(\left\langle\left. 0\right|_{E} \otimes\langle\psi|\right) U^{\dagger} U\left(|\phi\rangle \otimes|0\rangle_{E}\right)\right. \\
=\left(\left\langle\left. e\right|_{E} \otimes\langle\psi|\right)\left(|\phi\rangle \otimes|f\rangle_{E}\right)=\langle\psi \mid \phi\rangle(\langle e \mid f\rangle)_{E}\right.
\end{gathered}
$$

Hence, for $\langle\psi \mid \phi\rangle \neq 0$, we have $\langle e \mid f\rangle=1$, and therefore since the states are normalized, $|e\rangle=|f\rangle$. This means that no measurement in $\mathcal{H}_{E}$ can reveal any information that distinguishes $|\phi\rangle$ from $|\psi\rangle$.

Orthogonal quantum states (like classical information) can be reliably copied. For example, the unitary transformation that acts as

$$
\begin{aligned}
U: \quad|0\rangle_{A} \otimes|0\rangle_{B} & \longrightarrow \quad|0\rangle_{A} \otimes|0\rangle_{B} \\
|1\rangle_{A} \otimes|0\rangle_{B} & \longrightarrow \quad|1\rangle_{A} \otimes|1\rangle_{B},
\end{aligned}
$$

copies the first qubit onto the second if the first qubit is in one of the states $|0\rangle_{A}$ and $|0\rangle_{B}$. But if instead the first qubit is in the state $|\psi\rangle=a|0\rangle+b|1\rangle$, then

$$
U:\left(a|0\rangle_{A}+b|1\rangle_{A}\right)|0\rangle_{B} \quad \longrightarrow \quad a|0\rangle_{A}|0\rangle_{B}+b|1\rangle_{A}|1\rangle_{B}
$$

Thus is not the state $|\psi\rangle \otimes|\psi\rangle$.

To consider the most general possible quantum Xerox machine, we allow the full Hilbert space to be larger than the tensor product of the space of the original and the space of the copy. Then the most general copying unitary transformation acts as

$$
\begin{array}{cc}
U: \quad|\psi\rangle_{A}|0\rangle_{B}|0\rangle_{E} \longrightarrow \quad|\psi\rangle_{A}|\psi\rangle_{B}|e\rangle_{E} \\
|\phi\rangle_{A}|0\rangle_{B}|0\rangle_{E} \longrightarrow \quad|\phi\rangle_{A}|\phi\rangle_{B}|f\rangle_{E}
\end{array}
$$

Unitarity then implies that

$$
\langle\psi \mid \phi\rangle_{A}=\langle\psi \mid \phi\rangle_{A}\langle\psi \mid \phi\rangle_{B}\langle e \mid f\rangle_{E} ;
$$

therefore, if

$$
\langle\psi \mid \phi\rangle \neq 0
$$

then

$$
\langle\psi \mid \phi\rangle(\langle e \mid f\rangle)_{E}=1
$$

Since the states are normalized, we conclude that

$$
|\langle\psi \mid \phi\rangle|
$$

so that $|\psi\rangle$ and $|\phi\rangle$ actually represent thesame ray. No unitary machine can make a copy of both $|\phi\rangle$ and $|\psi\rangle$ if $|\phi\rangle$ and $|\psi\rangle$ are distinct, nonorthogonal states. This result is called the no-cloning theorem. [45] On the other it has been shown that if one uses non-unitary cloning devices, the cloning of non-orthogonal pure states remains impossible unless one is willing to tolerate a finite loss of fidelity in the copied states.

\section{7 conclusion}

The first step in cell division (mitosis or meiosis) is to make a copy of the DNA, a process called replication. In this process, DNA splits into two strands and each separated strand serves as a template for the construction of a new complementary strand and a copy of the genetic information present in the cells DNA. On the other hand we expect DNA may have quantum properties (such as quantum no-cloning theory). Then, according to the quantum no-cloning theorem, we have claimed that in each cell division we expect DNA inherit a near-exact (information in DNA) but not perfect copy of their parents. So in DNA-replication, information in DNA is not same and cells are not same [19]. Consequently, a cell in right limb is different from a cell in left limb and the act of replicating DNA is not quite perfect, and there are always small glitches in the copying process. Also when a new plant is formed from a cutting, due to the no cloning theorem we have different leaf structures and arrangements. Then it results from the no cloning theorem, the baby doesn't have the same DNA of the mother and doesn't have the same DNA as the father, the baby has its own DNA, unique and individual. A cloned creature, however, receives DNA from only one parent and we know according to the no-cloning theorem it is not possible to obtain a perfect copy of DNA (informationally) and in this process we have a finite loss of fidelity in the copies states [46]. Then all cloned animals are biologically flawed $[41,42,43,44]$.

Information is encoded by molecules, which are definitely quantum systems; but it is encoded in the nature of the molecules (adenine, thymine, cytosine, guanine), not in their state then it seems such an encoding is classical but this does not necessarily imply that the way Nature processes this information is entirely classical: this point is an open question [53].

\section{REFERENCES}

[1] P. Loi, C. Galli and G. Ptak. 2007. TRENDS in Biotechnology. doi:10.1016/j.tibtech.2007.03.007. 
[2] Louis-Marie Houdebine. 2003. Animal Transgenesis and Cloning. John Wiley and Sons.

[3] Arlene Judith Klotzko. 2001. The Cloning Sourcebook. Axford university Press.

[4] Heidi Ledford. 2007. Nature 445, 22.

[5] W. M. Rideout, K. Eggan and R. Jaenisch. 2001. SCIENCE. 293, 1098.

[6] Alan Colman and Justine Burley. 2007. Nature 447, 649-650.

[7] http://www.wi.mit.edu/news/archives/2006/rjo $117 . h t m l$

[8] Schrodinger, What is life? Cambridge University Press, London, 1934.

[9] R. Penrose, Shadows of the Mind Oxford University Press: 1994.

[10] P. C. W. Davies, 2004, 0.5em minus 0.4emBioSystems, 78,69 .

[11] G. Ch. Sirakoulis an I. Karafyllidis and Sandaltzopoulos, 2004, BioSystems 77, 11, 2004.

[12] W. H. Zurek, 2000, 0.5em minus 0.4emNature, 404, 9.

[13] J. McFadden, 2000, 0.5em minus 0.4emLondon.

[14] H. Frolich, 1970, 0.5em minus 0.4emNature 228, 1093.

[15] D. Home and R. Chattopadhyaya, 1996, 0.5em minus 0.4emPhys. Rev. Lett. 76, 2836-2839.

[16] A. Patel, 2001, 0.5em minus 0.4emJ. of Bioscience 26, 145-151.

[17] O. Nairz and M. Arndt and Zeilinger, 2003, 0.5em minus 0.4emAm. J. Phys., 71, 4.

[18] J. J. McFadden and Al-Khalili, 2003, Biosystems 50, 203-211.

[19] M. Mirzaee and M. Falahat chian, 2007, DNA and quantum theory, icqnm, p. 4, First International Conference on Quantum, Nano, and Micro Technologies (ICQNM).

[20] J. McFadden and J. Al-Khalili. 1999, 50(3):203211.

[21] A. Patel. 2003, In Computing and Information Sciences: Recent Trends, pages 271294. J.C.Misra, Narosa Publishing House.

[22] R. Penrose. 1989, The emperors new mind. The Clarendon Press Oxford University Press, New York, .

[23] Philip Cohen. 2001. NEW SCIENTIST, Vol. 169 No. 2276.

[24] D. N. Wells. 2005, Rev. sci. tech. Off. int. Epiz., 24 (1), 251-264.

[25] John Whitfield, Imprinting Marks Clones For Death, NATURE SCIENCE UPDATE, at http://www.nature.com/nsu/ 010712/010712-1.html

[26] Nancy Touchette, Cloned Mice Have Genetic Flaws, Genome news network Online, at http://gnn.tigr.org/articles $/ 09_{0} 2 /$ cloned.shtml (last visited Oct. 25, 2003).
[27] Ian Wilmut, Developmental Plasticity in Mammals: Implications For Biology and Medicine, Roslin Institute, at http://roslin.ac.uk/publications/9900annrep/plasticity.html (last visited Oct. 25, 2003).

[28] Harry Griffin, Should Assistance Dogs Be Cloned? Roslin Institute, at http://www.roslin.ac.uk/public/1208-02- cd.html.

[29] John Whitfield, Obituary: Dolly the Sheep, NATURE SCIENCE UPDATE (2003) at http://www.nature.com/nsu/ 030210/030210-15.htm.

l. [30] Adrienne N. Calhoun. 2004. bepress Legal Series http://law.bepress.com/expresso/eps/129.

[31] Dieter Egli1, Jacqueline Rosains1, Garrett Birkhoff1 and Kevin Eggan1. 2007. Nature, 447, 7.

[32] Xiangzhong Yang1, X Cindy Tian1, Chikara Kubota, Ray Page, Jie Xu, Jose Cibelli and George Seidel. Nature, doi:10.1038/nbt1276.

[33] Lefebvre, L., Viville, S., Barton, S. C., Ishino, F., Keverne, E. B., and Surani, M. A. (1998). Nat. Genet. 20, 163169 .

[34] Jose cibelli, Robert Lanza, Keith Campbell and Michael West. Principles of cloning. 2006 copyrighted material.

[35] Home, D., and Chattopadhyaya, R., 1996. Phys. Rev. Lett. 76. 2836-2839.

[36] Patel, A., 2001. J. of Bioscience 26, 145-151.

[37] A. J.F. Griffiths, S. R. Wessler, R. C. Lewontin, W. M. Gelbart, D. T. Suzuki and J. H. Miller. 2005. An introduction to genetic analysis. W. H. Freeman Publishers.

[38] W.K.Wootters and W.H. Zurek, 1982, Nature 299, 802.

[39] Lewis, and Ricki., 2001, Human Genetics concepts and Application, Macgraw Hill, P. 124.

[40] D.V.Sych, B.A.Grishanin, V.N.Zadkov, 2006, Laser Phys. Lett., 3(2), 102.

[41] Hochedlinger, K., and Jaenisch, R., 2003, The new england journal of medicine, ;349, 275-86.

[42] www.wi.mit.edu/news/archives/2006/rj0117.html

[43] www.mindfully.org/GE/GE4/all-cloned-genedefects28apr02.html

[44] Lodish, H., Berk, A., Matsudaira, P., Kaiser, M., Scott, M. P., Zipursky, S. L and Darnell, J., Molecular Cell Biology, W. H. Freeman and Company, New York, 2004.

[45] Preskill, Quantum information (lecture notes).

[46] M. A. Nielsen and I. L. Chuang, Quantum Computation and Quantum Information, Cambridge University Press, 2000.

[47] www.tamiu.edu/student/journalism/Vol3No1.html

[48] Nikolaenko, N. N., 2003. Artistic thinking and cerebral asymmetry, acta neuropsy chologica. 1, 1 .

[49] Baker, A. H., and Ledner, A. I. 2004. Journal of General Psychology. 131(1),36.

[50] Hamada, P.A., and Yokoyama, T. 1998. Nature Biol. 210, 277287. 395, 177. 
[51] Varlet, I. and Robertson, E.J., 1997. Curr Opin Genet Dev. 7(4), 519.

[52] Housman, D., 1995. Molecular medicine, 332, 5.
[53] V. Scarani, S. Iblisdir, N. Gisin and A. Acin. 2005. Rev. Mod. Phys. 77, 1225-1256. 